TRABAJOS DE PREHISTORIA

78 N N. $^{\circ}$ 1, enero-junio 2021, pp. 153-163, ISSN: 0082-5638

https://doi.org/10.3989/tp.2021.12270

\title{
Arte paleolítico en Salamanca. Nuevos hallazgos en el yacimiento de EI Paraje de La Salud ${ }^{*}$
}

\author{
Palaeolithic rock art in Salamanca. New findings in El Paraje de La Salud site
}

\author{
Olivia Rivero ${ }^{\mathrm{a}}$, Julián Bécares ${ }^{\mathrm{a}}$ y Esteban Álvarez-Fernández ${ }^{\mathrm{a}}$
}

\section{RESUMEN}

En este trabajo se presentan los resultados de la intervención realizada en el yacimiento de El Paraje de La Salud (Salamanca), con motivo del descubrimiento de un nuevo panel con grabados de cronología paleolítica. El conjunto ha sido estudiado empleando técnicas de restitución tridimensional con el fin de documentar tanto el soporte como los motivos grabados. Se trata de una muestra más de arte con cronología premagdaleniense realizado al aire libre, cuyas características técnicas y formales permiten ponerlo en relación con representaciones aparecidas en los conjuntos portugueses de Fariseu y Penascosa en el Côa, así como con el recientemente descubierto en Foz do Río Tua.

\begin{abstract}
This paper presents a study of the newly discovered panel with Palaeolithic engravings at El Paraje de La Salud (Salamanca). We use three-dimensional restitution techniques in order to document both the support and the recorded motifs. It is one more example of open-air Premagdalenian art. Their technical and formal characteristics are similar to those of representations at the Portuguese sites of Fariseu and Penascosa in the Côa, as well as those at the recently discovered Foz do Río Tua.
\end{abstract}

Palabras clave: Paleolítico Superior; península ibérica; arte al aire libre; fotogrametría de objeto cercano; piqueteado; animación; Premagdaleniense.
Key words: Upper Paleolithic; open air rock-art; close-range photogrammetry; pecked technik; animation; Premagdalenian.

\section{INTRODUCCIÓN}

El arte paleolítico del interior es conocido desde los comienzos de la historia de la disciplina en la península ibérica, con los descubrimientos de Penches (Burgos) (Hernández Pacheco 1917), Los Casares y La Hoz (Guadalajara) (Cabré 1934), Maltravieso (Cáceres) (Callejo 1958), El Reguerillo (Madrid) (Hernández Pacheco 1959: 261), Domingo García (Gonzalo Quintanilla 1970; Lucas Pellicer 1973) y La Griega (Almagro Gorbea 1971). Sin embargo, estas manifestaciones artísticas no han adquirido personalidad propia hasta la década de los 80 , con el descubrimiento y estudio de los sitios con arte al aire libre de Mazouco (Freixo de Espada à Cinta, Portugal) (Jorge et al. 1981, 1982), Piedras Blancas en Almería (Martínez García 1986-1987) y Fornols-Haut en los Pirineos orientales franceses (Sacchi et al. 1988). A partir de los 90 el hallazgo de los conjuntos al aire libre de Domingo García (Segovia) (Ripoll y Municio 1999), Siega Verde (Salamanca) (Balbín et al. 1991; Alcolea y Balbín 2006, 2012) y Foz Côa (Vila Nova de Foz Côa), acaban convirtiendo actualmente la península en un punto de referencia en los estudios sobre arte paleolítico en Europa (Baptista 2009).

A este cada vez más dilatado conjunto de representaciones rupestres al aire libre se han sumado a los de Alto Sabor, Ribeira da Sardinha, Zézere y Ocre-

\footnotetext{
* Este trabajo se enmarca en el proyecto de investigación “Aprendizaje y desarrollo de las capacidades artísticas en los Humanos Anatómicamente Modernos; un enfoque multidisciplinar” (HAR2017-87739-P), financiado por el Ministerio de Ciencia, Innovación y Universidades. IP: Olivia Rivero

a Universidad de Salamanca, Dept. de Prehistoria, Historia Antigua y Arqueología (GIR PREHUSAL). C/ Cerrada de Serranos s/n. 37002 Salamanca. España. Correos e: OR oliviariver@usal.es https://orcid.org/0000-0002-0730-4854; JB jbecares@usal.es https://orcid.org/0000-0003-4697-1737; EAF epanik@usal.es https://orcid.org/0000-0002-7895-3421

Recibido 10-XI-2020; aceptado 14-I-2021.
} de uso y distribución "Creative Commons Reconocimiento 4.0 Internacional" (CC BY 4.0) 
za (Baptista 2009), Redor do Porco (Baptista y Reis 2011), Arroyo de las Almas (Reis y Vázquez 2015) y el abrigo de Foz do Río Tua (Teixeira y Sanches 2017), así como los motivos paleolíticos en el yacimiento de El Paraje de La Salud, en Salamanca (Garate et al. 2016), conjuntos revisados recientemente en un trabajo de síntesis (Santos 2019). Por otra parte, destaca el hallazgo de un extenso conjunto de arte mobiliar en el yacimiento de Foz do Medal en el valle del Sabor (Portugal) (Figueiredo et al. 2014).

En el año 2018, en el yacimiento de El Paraje de la Salud se han descubierto estas nuevas figuras que nos ocupan. Están próximas a las ya conocidas y vienen a completar y a enriquecer nuestro conocimiento del arte paleolítico de este conjunto, así como a resaltar sus vinculaciones con los sitios portugueses de Foz Côa y Foz do Río Tua.

\section{LOCALIZACIÓN DEL YACIMIENTO Y DE LOS NUEVOS DESCUBRIMIENTOS}

El conjunto de representaciones rupestres al aire libre de La Salud, descubierto en 2013, ha sido estudiado por un equipo de investigación encabezado por D. Garate y M. Santonja (Garate et al. 2016). Se localiza en ambas márgenes del río Tormes, muy cerca de la ciudad de Salamanca y en su mismo término municipal. Ahora se añade un pequeño grupo de grabados, realizados mediante piqueteado e incisión (Figs. 1 y 2).

A inicios de 2018 D. Francisco Guijarro y Dña. Carmen Centeno comunicaron a uno de nosotros (J. B.) la existencia de una figura de caballo en El Paraje de La Salud. Este descubrimiento fue comunicado a la Arqueóloga Territorial, Dña. Esther González Mazariego, y tras la correspondiente autorización, se analizó y documentó el panel que aquí presentamos.

Se localiza en un abrigo muy próximo al cauce actual del río en su margen izquierda. El nivel actual del agua, retenida por la presa de El Marín, está a menos de un metro del arranque de la pared que sirve de soporte a los grabados. Está $60 \mathrm{~m}$ aguas arriba de la desembocadura de un barranco que delimita por el sureste un promontorio donde se abren los túneles de un antiguo polvorín, y a $260 \mathrm{~m}$ en línea recta del conjunto S2, ya publicado (Garate et al. 2016: 22-24). Se accede a él por un corto, estrecho y pendiente barranco y sus coordenadas son $40^{\circ} 57^{\prime} 33^{\prime \prime}$ de latitud norte y $05^{\circ} 42^{\prime} 38^{\prime \prime}$ de longitud oeste de Greenwich.

El abrigo, en líneas generales, está compuesto por una superficie central, dos salientes laterales y una pequeña visera de unos $80 \mathrm{~cm}$ que le confieren una relativa protección. La superficie de su parte central mide unos $3 \mathrm{~m}$ de ancho, comprendidos entre los dos salientes rocosos. El saliente a su izquierda sobresale unos 2

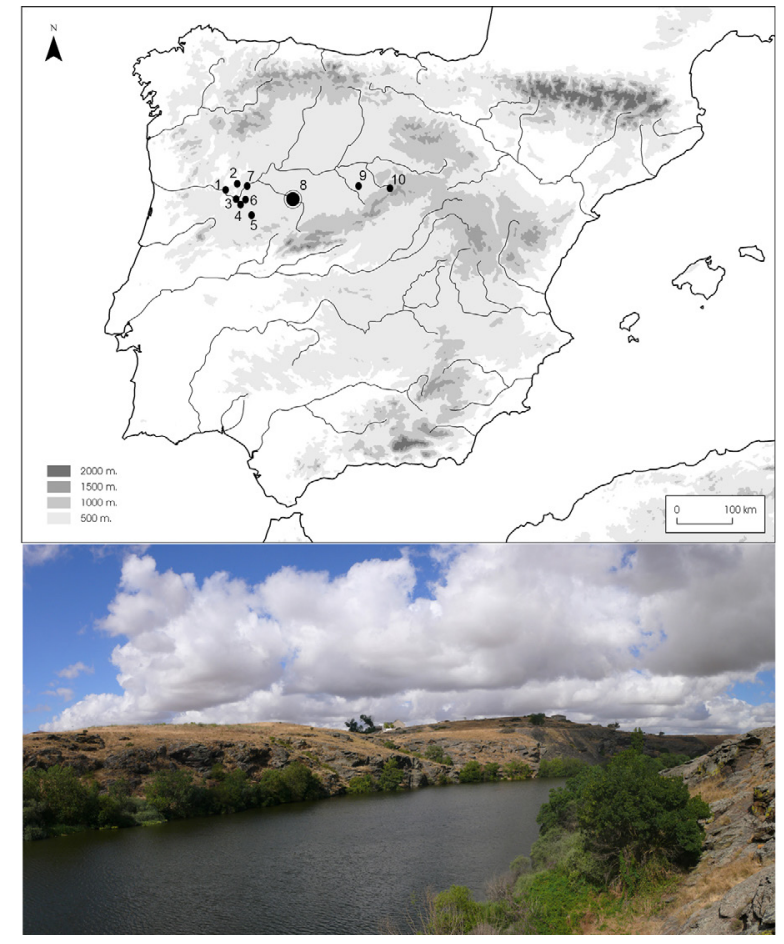

Fig. 1. Localización de El Paraje de La Salud y de los sitios de arte paleolítico del valle del Duero mencionados en el texto. 1. Foz da Río Túa (Castanheiro). 2. Foz do Medal (Tras-os-Montes). 3. Conjunto de yacimientos de Foz Côa (Vila Nova de Foz Coa). 4. Redor do Porco (Es-calhão, Figueira de Castelo Rodrigo). 5. Siega Verde (Salamanca). 6. Arroyo de las Almas (Salamanca). 8. La Salud (Salamanca). 9. Conjunto de Domingo García (Segovia). 10. La Griega (Segovia). Fotografía de El Paraje de La Salud, enclave en el que se localizan los nuevos grabados localizados en 2018 (fotografía J. Bécares). En color en la edición electrónica.

m en perpendicular a la misma y es más irregular: El situado a su derecha, algo más oblicuo, apenas sobresale $1 \mathrm{~m}$.

Esta superficie central es irregular hasta aproximadamente $1 \mathrm{~m}$ del suelo, y algo más lisa a partir de este punto, así como bastante vertical (entre $80^{\circ}$ y $85^{\circ}$ ), estando orientada al noreste. Está surcada horizontalmente, en su parte central, por una grieta que se ensancha y abre en $\mathrm{Y}$ en el sector derecho. Otras fisuras menores lo cruzan verticalmente. La más ancha y profunda, situada algo a la derecha de la parte inferior de la superficie central, delimita un espacio parcialmente liso de aproximadamente un metro de ancho por $50 \mathrm{~cm}$ de alto, en el que se localizan los grabados que nos ocupan.

El resto de la superficie central, así como parte de los salientes, presentan espacios lisos similares, o incluso con mejores condiciones para soportar grabados. La presencia de abundantes líquenes nos ha impedido apreciarlos por el momento (Fig. 3). 


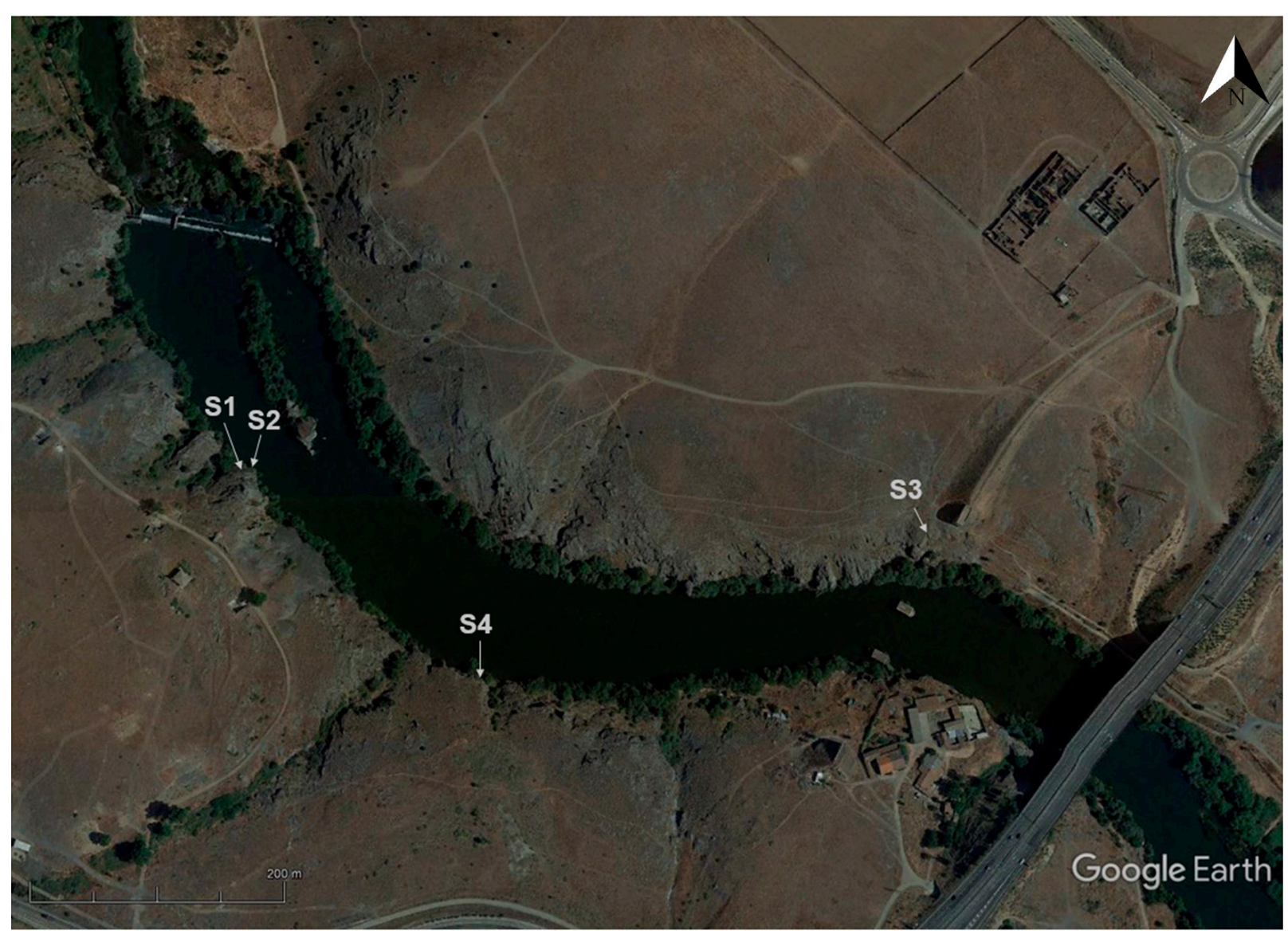

Fig. 2. Situación de los 4 conjuntos de grabados paleolíticos localizados hasta ahora en El Paraje de La Salud (Salamanca). El conjunto motivo de esta noticia está señalado en el mapa como S4. En color en la edición electrónica.

La roca soporte no difiere de la ya señalada: pizarras grises y negras del Ordovícico, según la hoja 478 del Mapa Geológico 1:50.000 del Instituto Geológico y Minero de España en su edición de 1993 (Garate et al. 2016).

\section{METODOLOGÍA DE ESTUDIO}

La metodología de documentación gráfica empleada ha sido la fotogrametría de objeto cercano, ya que, al tratarse de un único panel decorado, era la más apropiada para documentar tanto el soporte, como los grabados. Se parte de la aplicación de técnicas de restitución tridimensional (fotogrametría digital), combinada con sistemas de tratamiento digital y la restitución gráfica. Esta combinación de técnicas $3 \mathrm{D}$ y $2 \mathrm{D}$ es hoy en día habitual en el estudio y restitución del arte prehistórico (Domingo et al. 2013; Plisson y Zotkina 2015; Fritz et al. 2016).
En los últimos años, el registro 3D del volumen de los sitios con arte paleolítico se ha convertido en la metodología estándar (e.g. Bourdier et al. 2008; Azéma et al. 2010; Feruglio et al. 2013; Garate et al. 2013; Garate y Rivero 2015; Robert et al. 2016). En este trabajo se han empleado técnicas de fotogrametría de objeto cercano basadas en tomas fotográficas no ordenadas y herramientas de visión artificial o computer vision, basadas en un algoritmo de transformación de característica en escala invariable (Scale-invariant feature transform o algorimo SIFT por sus siglas en inglés). Así, se han elaborado modelos 3D con iluminación natural y con iluminación rasante lograda mediante el empleo de flashes externos. Estos últimos modelos están destinados a facilitar la identificación del grabado con vistas a su posterior restitución. Esta metodología ha sido implementada para facilitar tanto la reproducción del soporte como la visibilidad del grabado (Rivero et al. 2019). 

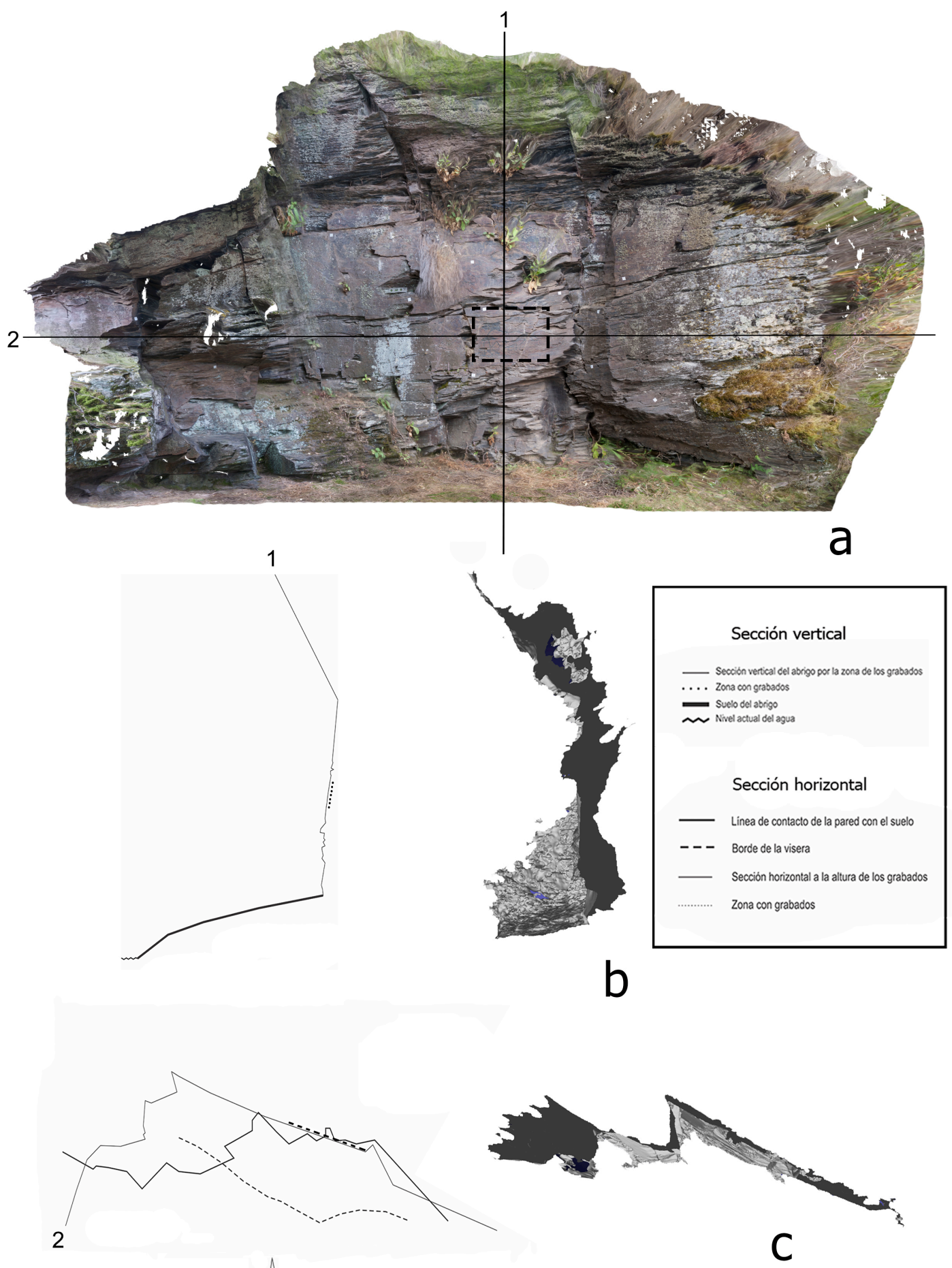

$1 \mathrm{~m}-\bigcup_{\text {N.M. }}$

Fig. 3. Abrigo de La Salud: a. Modelo fotogramétrico general del abrigo; b. Alzado del abrigo; c. Planta y secciones de la visera y de la zona correspondiente a los grabados. Modelo fotogramétrico O. Rivero y J. Bécares. En color en la edición electrónica. 
La interpretación de los registros gráficos se ha llevado a cabo directamente sobre los productos derivados de los modelos 3D obtenidos. Los motivos identificados a partir de procedimientos habituales de tratamiento de imagen, usados en los últimos años para la generación de calcos digitales, han sido integrados de modo directo en las restituciones 3D de los paneles estudiados. Dada la resolución de los modelos $3 \mathrm{D}$, la precisión del registro supera la propia de un calco directo. Una vez acopladas las diversas texturas, en la versión final los trazos grabados recuperan su conexión con el soporte, respetando la relación espacial con la superficie para la que se concibieron y sobre la que se ejecutaron, sin distorsiones ni respecto a su ubicación, ni a su relación con el resto de los motivos conservados en el panel.

\section{IDENTIFICACIÓN DE LAS NUEVAS EVIDENCIAS}

Se han identificado tres motivos de équidos (SA.I.1. a 3.), definidos mediante las técnicas de piqueteado y grabado inciso (Tab. 1). Dos de ellos conforman un caballo bicéfalo orientado a la derecha. El tercero es una línea cérvico-dorsal situada a la derecha de la figura completa, probablemente afrontada, aunque no se puede saber con exactitud al tratarse de un motivo incompleto. Las figuras aparecen a una altura de $141 \mathrm{~cm}$ sobre el nivel del suelo actual (Fig. 4).

SA.I.1. Caballo orientado a la derecha, grabado mediante piqueteado e incisión. La cabeza, orientada hacia abajo, tiene el hocico indicado con el característico "pico de pato", oreja, crinera lineal con escalón, línea cérvico-dorsal con la cruz marcada, cola más larga que las patas traseras, pata trasera, vientre y doble pata delantera en perspectiva frontal, así como línea de pecho (Fig. 5c).

\begin{tabular}{|c|c|c|c|}
\hline Sigla & Motivo & Técnica & Dimensiones \\
\hline SA.I.1. & Caballo & $\begin{array}{c}\text { Piqueteado y } \\
\text { grabado }\end{array}$ & $55.5 \times 25.5 \mathrm{~cm}$ \\
\hline SA.I.2. & Caballo & $\begin{array}{c}\text { Piqueteado y } \\
\text { grabado }\end{array}$ & $50 \times 33.5 \mathrm{~cm}$ \\
\hline SA.I.3. & $\begin{array}{c}\text { Posible } \\
\text { caballo }\end{array}$ & Piqueteado & $24 \mathrm{~cm}$ \\
\hline
\end{tabular}

Tab. 1. Inventario de motivos identificados en 2018 en El Paraje de La Salud (Salamanca).

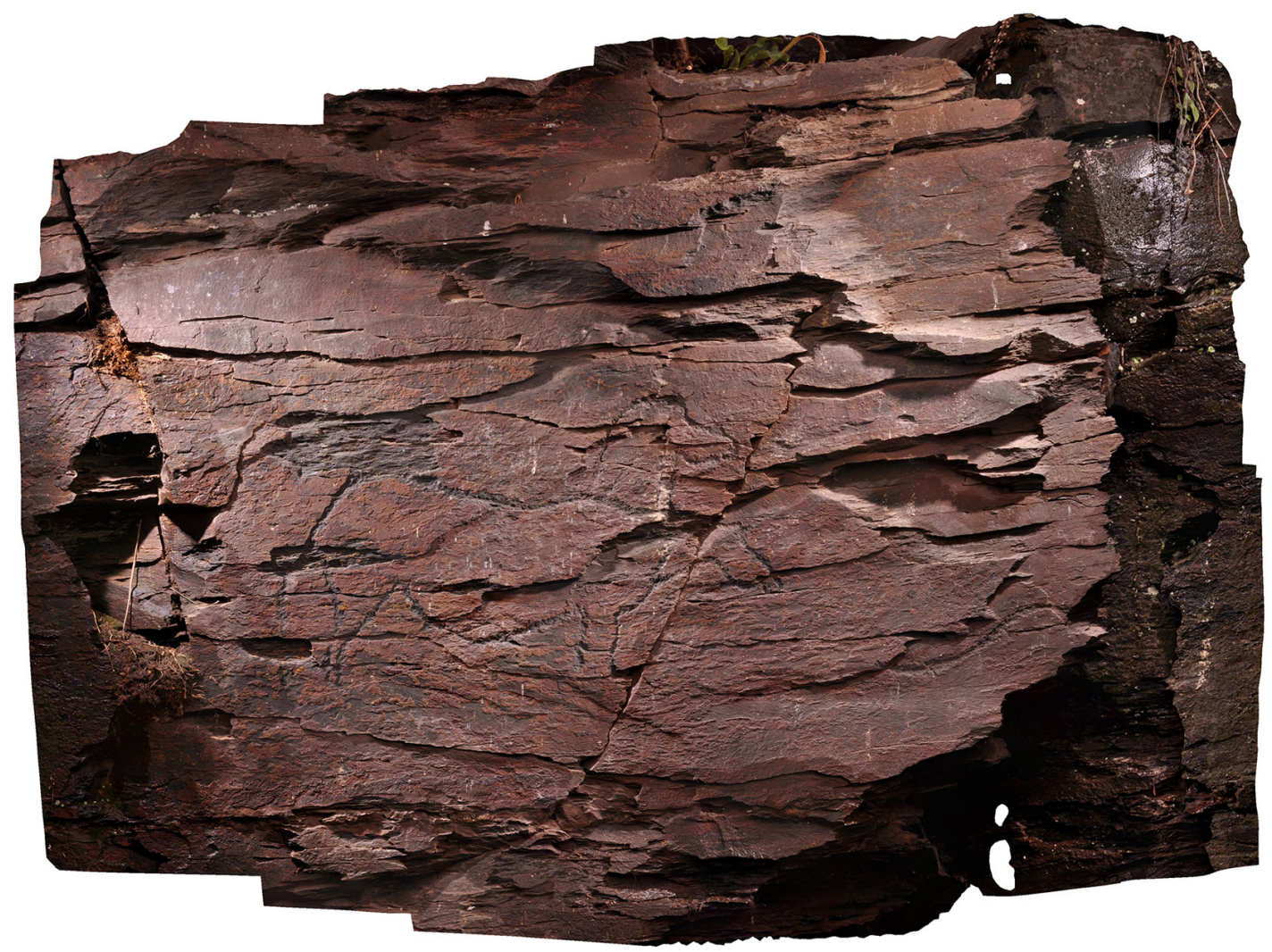

Fig. 4. Ortoimagen del panel decorado donde se aprecian los motivos grabados. Ortofoto O. Rivero. En color en la edición electrónica. 

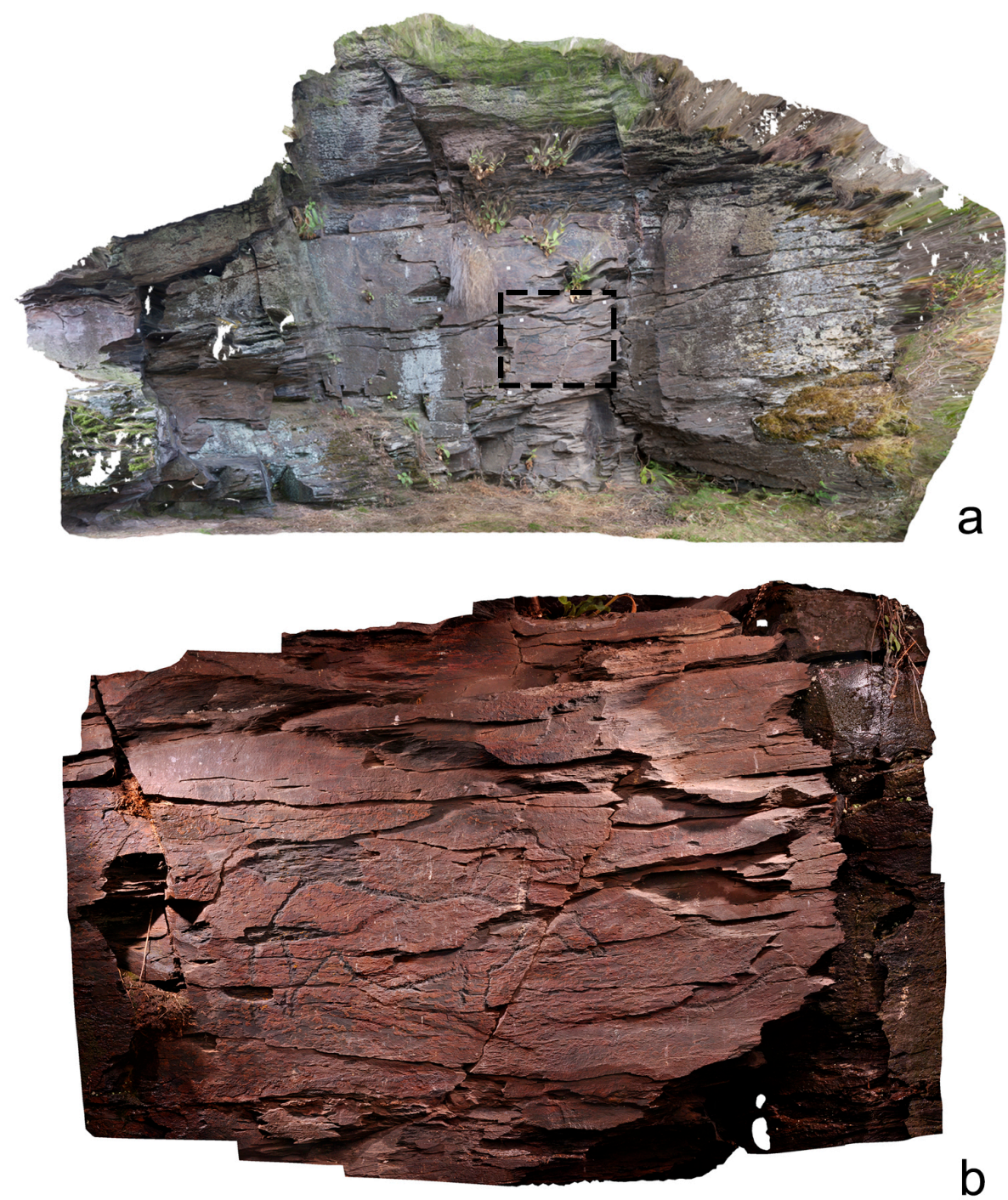

b

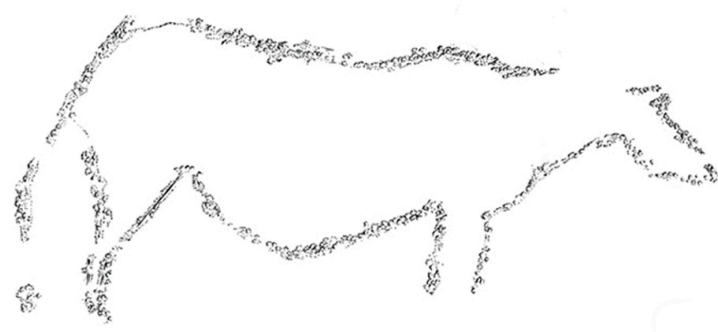

C

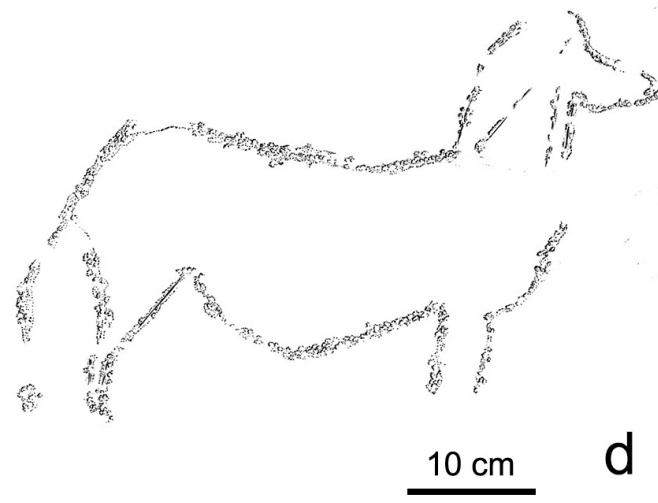

Fig. 5. a. Modelo fotogramétrico general del abrigo, con la localización del panel grabado. b. Ortoimagen del panel decorado. c. Calco del caballo SA.I.1. d. Calco del caballo piqueteado SA.I.2. Fotogrametría O. Rivero y J. Bécares. Ortoimagen y calcos de los motivos O. Rivero. En color en la edición electrónica (salvo calcos). 

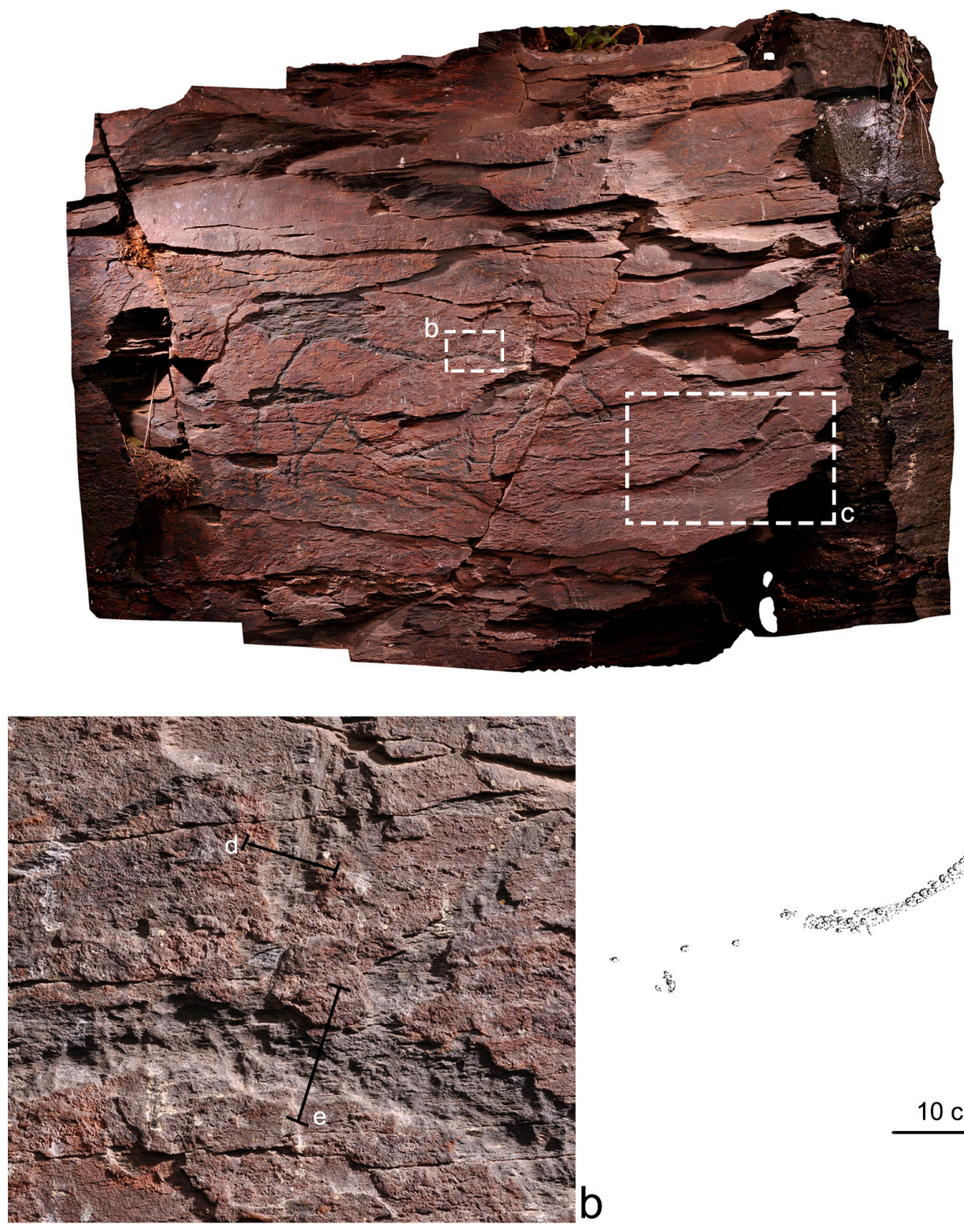

a
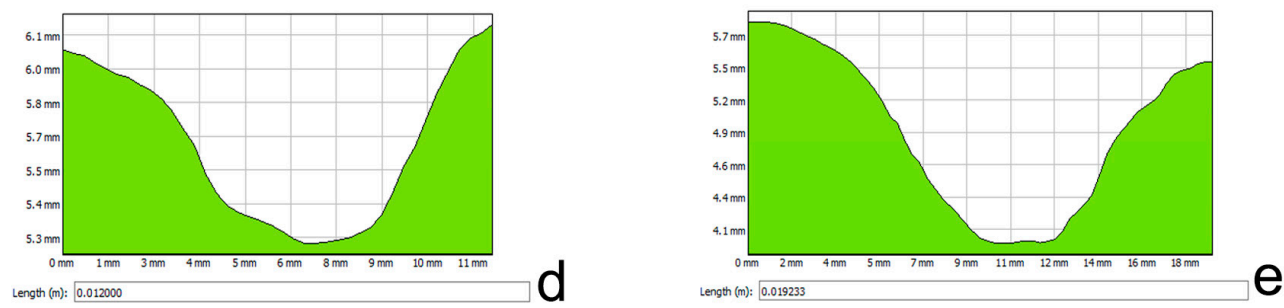

Fig. 6. a. Ortoimagen del panel decorado. b. Detalle del piqueteado en el caballo SA.I.1. (cruz) y en el caballo SA.I.2. (final de la crinera). En el segundo se aprecia la menor profundidad del piqueteado y la incisión que repasa parcialmente la línea exterior de la crinera. c. Calco del caballo piqueteado SA.I.3. d. Perfil del trazo correspondiente a la crinera del caballo SA.I.2. e. Perfil del trazo de la cruz del caballo SA.I.1. Puede apreciarse la diferencia de anchura y profundidad entre ambos antes señalada. Ortoimagen, foto y calco O. Rivero. 
La cadena operativa consiste en el piqueteado total del motivo. Además la línea interior de la pata trasera ha sido repasada mediante incisión. La pata delantera derecha ha sido realizada mediante una única hilera de golpes y la izquierda mediante doble hilera de piqueteado efectuado de arriba abajo. No podemos saber con certeza la superposición o infraposición con respecto a la figura SA.I.2., si bien en el caso del caballo SA.I.1. se encuentra piqueteado con más profundidad y probablemente es la primera figura que se realizó.

SA.I.2. Caballo orientado a la derecha, grabado mediante piqueteado e incisión. La cabeza está levantada con el hocico indicado en el característico "pico de pato". Tiene crinera doble lineal con escalón, y doble línea de pecho. El resto de la figura corresponde al caballo SA.I.1. (Fig. 5d).

Este tipo de representación se relaciona con un probable intento de expresar la animación de la figura por su descomposición en partes, tal y como ha sido definido por M. Azema (1992a y 1992b) y L. Luís (2012). En concreto, según la clasificación de este último, se trataría de una animación por descomposición y superposición segmentaria, que afecta en este caso solo al prótomo del animal (cabeza y pecho).

Según la cadena operativa, esta figura está menos profundizada que la SA.I.1. Las dos líneas de la crinera han sido repasadas mediante incisión. Asimismo, posee un despiece interno en el pecho que se interrumpe a la altura de la línea cérvico-dorsal de SA.I.1. Ello nos lleva a considerar que posiblemente se hiciera en segundo lugar (Fig. 6), aunque no podemos establecer con seguridad el orden de ejecución de las figuras.

SA.I.3. Caballo orientado a la izquierda, grabado mediante piqueteado. Como únicamente se ha representado un fragmento de línea cérvico-dorsal, no se puede saber con certeza si la figura se orienta hacia la derecha o hacia la izquierda. No obstante la incurvación del extremo derecho de la línea sugiere la forma de las nalgas del animal (Fig. 6c).

\section{RESULTADOS}

La metodología expuesta más arriba ha permitido documentar con gran fidelidad los motivos descubiertos en el nuevo panel de arte rupestre del conjunto de La Salud. Sus tres representaciones de caballos (Fig. 7) están grabadas mediante piqueteado e incisión ocasional. Sus características permiten relacionarlas por su temática, técnica, soporte y convenciones estilísticas con otros conjuntos de arte paleolítico al aire libre como Domingo García (Segovia) o las representaciones piqueteadas de Foz Côa. Nos fijamos, p. ej., en la representación de las patas delanteras en perspectiva frontal (Guy 2002) y, más en concreto, en las de caba- llos animados mediante descomposición por yuxtaposición segmentaria de las rocas 4 de Penascosa (Baptista 2009; cf. número 07, según la clasificación de Santos 2019) y 1 Fariseu en el Côa ( $n^{\circ} 45$ y 61 según Santos 2019), así como en la roca 1 de Canada do Inferno (figura 11 según Santos 2019). En Foz do Río Tua se trata de dos especies distintas, ciervo y caballo (Teixeira y Sanches 2017).

La cronología de estas representaciones, a partir de las dataciones por superposición de la estratigrafía en la roca 1 de Fariseu, se estima anterior a la fecha del nivel 8, datado en $18400 \pm 1600$ BP (Baptista 2012: 12; Aubry et al. 2014). Según los investigadores de este yacimiento, el suelo contemporáneo de los grabados habría desaparecido por un proceso erosivo y se situaría cronológicamente entre la fecha anterior y 19020 $\pm 80 \mathrm{BP}$, datación obtenida de una muestra recogida en un sondeo situado a pocos metros de la roca 11. El grabado de esta roca sería por lo tanto anterior a este episodio erosivo (Aubry et al. 2014) y se situaría en los límites del Gravetiense-Solutrense.

Este hecho coincide con las características formales de las figuras, en particular con los hocicos en pico de pato, la perspectiva frontal de las patas o las crineras lineales con escalón, que permiten poner en relación estas representaciones con otros conjuntos del arte del interior peninsular como La Griega (Corchón 1997), atribuibles a la fase premagdaleniense (Sauvet y Sauvet 1983; Balbín y Alcolea 1994; Rivero 2010; Corchón et al. 2012).

\section{CONCLUSIONES}

La noticia que publicamos aquí añade tres motivos al corpus de figuras representadas en el yacimiento de La Salud, así como permite aquilatar con mayor precisión su cronología y relación con otros conjuntos de arte paleolítico al aire libre del interior de la península ibérica.

Esta serie de descubrimientos puede ponerse en relación con la fase antigua del Côa y son la expresión de un poblamiento premagdaleniense, de probable cronología Gravetiense, en torno al valle del Duero: Possadouro, Sampaio, Ribeira da Sardinha y Fraga Escrevida, en el valle del río Sabor; Mazouco y Fraga do Gato, en el propio valle del Duero; así como Foz do Río Tua). Al sur del Duero pertenecerían a este mismo momento los sitios de Poço do Caldeirão y Costalta (Barroca), situados en valle del Zêzere, así como el caballo del valle del Ocreza (Baptista 2012).

Las características técnicas y formales de estas figuras sugieren un fuerte parentesco. Destacan el uso de la animación por descomposición y yuxtaposición segmentaria, la incisión/abrasión en la parte delantera del animal, amén de los paralelos formales ya mencio- 


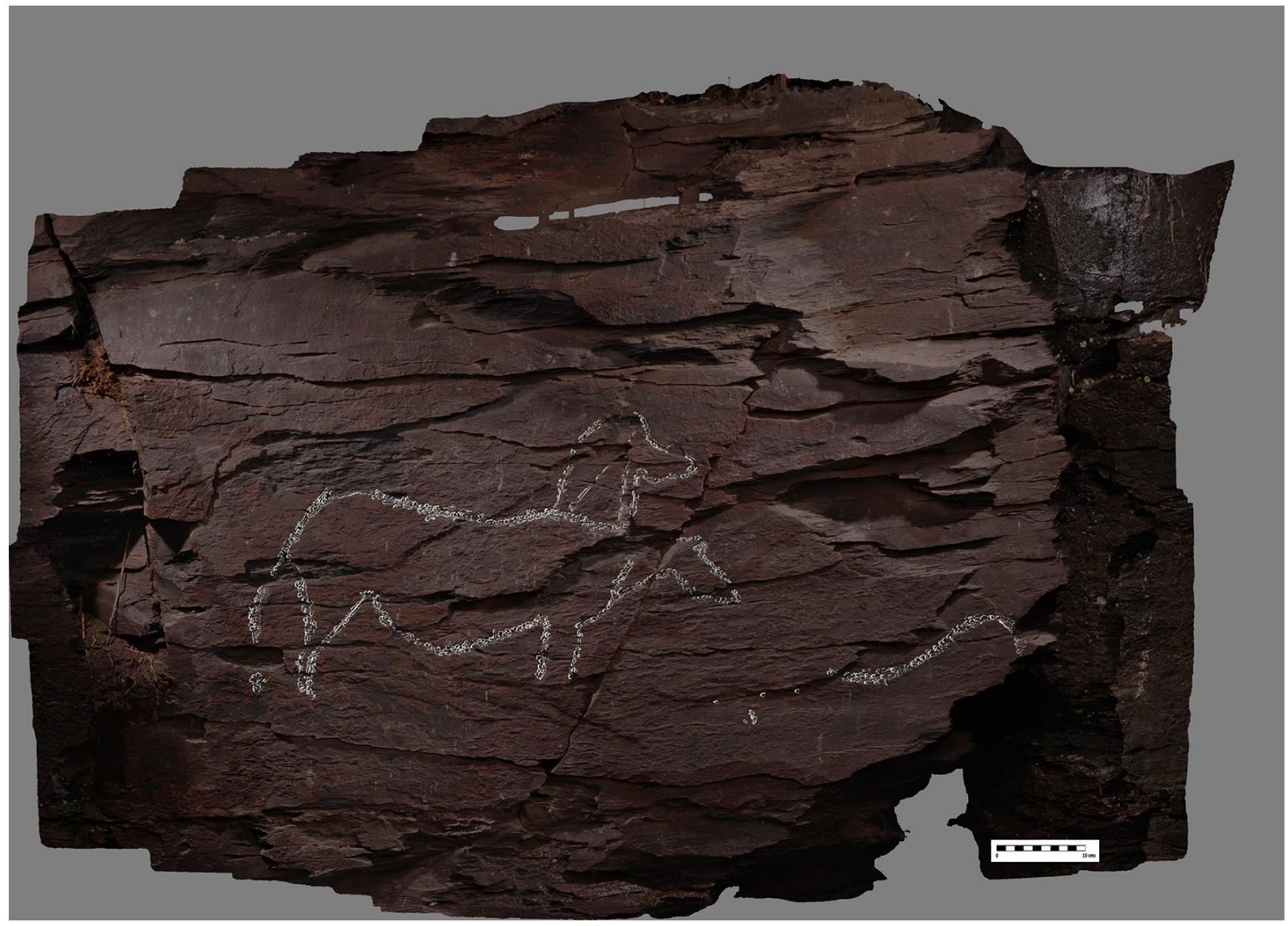

Fig. 7. Calco del panel decorado con los motivos identificados sobre ortoimagen. Documento O. Rivero. En color en la edición electrónica.

nados como la crinera en escalón. El conjunto ha llevado a plantear no solo la contemporaneidad sino incluso una posible autoría compartida para algunas de estas representaciones, según Baptista (2012). Para este investigador estos conjuntos con escaso número de representaciones que se sitúan en el área de influencia del Côa podrían funcionar como marcadores territoriales durante este período.

Los yacimientos de cronología Gravetiense conocidos actualmente en el valle del Duero se encuentran exclusivamente en el valle del Côa. Se trata de Olga Grande 4 y Cardina I, cuyas fechas de TL oscilan entre $31000 \pm 2500 \mathrm{BP}$ y $26800 \mathrm{r} \pm 2300 \mathrm{BP}$ para el primero y $30100 \pm 1500$ BP y $20700 \pm 1300$ BP para el segundo (Valladas et al. 2001).

Los sitios de Buraca Grande (con una fecha de $23920 \pm 300$ BP), Buraca Escura (datado en $21820 \pm$ $200 \mathrm{BP}$ ) y el yacimiento al aire libre de Vale das Buracas se sitúan en cronología Gravetiense (Aubry et al. 2012), pero están geográficamente alejados del núcleo del Duero. Los otros vestigios arqueológicos corres- pondientes a esta etapa son los del sitio al aire libre del Valle de las Orquídeas (Atapuerca), datado entre el 29000 y el 27000 BP (Díez y Navarro 2005).

El poblamiento premagdaleniense de la provincia de Salamanca está documentado de manera indirecta por el aprovisionamiento de las materias primas identificadas en el Valle del Côa durante el Gravetiense (Aubry et al. 2012). Los datos disponibles para los yacimientos de Olga Grande 4 y Cardina I muestran que las rocas silíceas en este período procedían de distintas áreas de la meseta (en torno a la localización actual de la ciudad de Salamanca principalmente) y la Estremadura portuguesa, sugiriendo una red de intercambios con estas dos regiones. Las figuras de La Salud aquí documentadas reforzarían esta hipótesis, puesto que sus fuertes paralelismos con las representaciones de Canada do Inferno y Fariseu son un ejemplo más de los vínculos culturales entre estas dos zonas durante el Gravetiense.

Estos datos vienen a completar el supuesto vacío poblacional de la meseta durante el Paleolítico Supe- 
rior. Muestran que, al contrario, existía un profundo conocimiento del entorno por parte de las poblaciones que lo habitaban, así como redes de intercambio a media y larga distancia que sugieren una continuidad y una mayor homogeneidad cultural de lo que se consideraba hace unas décadas (Corchón 2006).

Los recientes hallazgos en el yacimiento de $\mathrm{La}$ Salud vienen a enriquecer el conocimiento sobre este conjunto, cuyas características son de gran interés al mostrar una relación más estrecha con los conjuntos portugueses que con el yacimiento de Siega Verde, sitio con el que posee más proximidad geográfica.

Este hecho nos muestra que estos yacimientos participan en una red de relaciones a corta y media distancia cuya amplitud probablemente es superior a lo que se consideraba anteriormente, señalando un hábitat continuado en la meseta durante el Paleolítico Superior antiguo.

\section{BIBLIOGRAFÍA}

Alcolea, J. J. y Balbín, R. 2006: Arte Paleolítico al aire libre. El yacimiento rupestre de Siega Verde, Salamanca. Memorias Arqueología en Castilla y León 16, Junta de Castilla y León. Consejería de Cultura y Turismo. Valladolid.

Alcolea, J. J. y Balbín, R. 2012: "El Arte rupestre Paleolítico del interior peninsular". En Arte sin artistas. Una mirada al Paleolítico. Museo Arqueológico Regional. Alcalá de Henares: 184-207.

Almagro Gorbea, M. 1971: "La Cueva del Niño (Albacete) y la Cueva de la Griega (Segovia). Dos yacimientos de arte rupestre recientemente descubiertos en la Península Ibérica". Trabajos de Prehistoria 28: 9-62.

Aubry, T.; Luís. L.; Mangado, J. y Matias, H. 2012: "We will be known by the tracks we leave behind: Exotic lithic raw materials, mobility and social networking among the Côa Valley foragers (Portugal)". Journal of Anthropological Archaeology 31: 528-550. https://doi.org/10.1016/j.jaa.2012.05.003

Aubry, T.; Santos, A. y Luís, L. 2014: "Stratigraphies du panneau 1 de Fariseu: analyse structurelle d'un système graphique paléolithique à l'air libre de la vallée du Côa (Portugal)". Paleo, 2014, numéro spécial 'Micro-analyses et datations de l'art préhistorique dans son contexte archéologique': 259-270

Azema, M. 1992a: "La décomposition du mouvement dansl'art animalier paléolithique des Pyrénées". Préhistoire et Anthropologie Méditerranéennes 1: 17-31.

Azema, M. 1992b: "La représentation du mouvement dans 1'art animalier paléolithique des Pyrénées". Préhistoire Ariégeoise 47: 19-76.

Azéma, M.; Gély, B.; Prudhomme, F. y ATM 3D Société 2010: "Relevé 3D de gravures fines Paléolithiques dans l'abri du Colombier (Gorges de l'Ardèche)". En G. Piçon y J.-M. Geneste (eds.): Art Rupestre: la 3D, un outil du médiation de réel invisible?. In situ, Revue des Patrimoines 13: 1-14. https://doi.org/10.4000/insitu.6723

Balbín, R. de y Alcolea, J. 1994: “Arte paleolítico en la Meseta española”. Complutum 5: 97-138.

Balbín, R. de; Alcolea, J.; Santonja, M. y Pérez Martín, R. 1991: "Siega Verde (Salamanca). Yacimiento artístico paleolítico al aire libre". En M. Santonja (ed.): Del Paleolítico a la Historia. Junta de Castilla y León y Museo de Salamanca. Salamanca: 33-47.

Baptista, A. M. 2009: O paradigma perdido: O Vale do Côa e a arte paleolitica de ar livre em Portugal. Edições Afrontamento, Parque Arqueológico do Vale do Côa. Porto/Vila.
Baptista, A. M. 2012: "El arte paleolítico en Portugal". En I. Escobar y B. Rodríguez (eds.): Arte sin artistas: una mirada al Paleolítico. Ed. D-L. Madrid: 305-338.

Baptista, A. M. y Reis, M. 2011: “A rocha gravada de Redor do Porco. Um novo sítio com arte paleolítica de ar livre no rio Águeda (Escalhão, Figueira de Castelo Rodrigo)". Côa Visão 13: 15-20.

Bourdier C.; Fuentes, O.; Hamon, G. y Pinçon, G. 2008: "Technologies 3D appliquées à la sculpture pariétale magdalénienne". En O. Buchsenschutz (ed.): Images et relevés archéologiques, de la preuve à la démonstration. $132^{e}$ Congrès CTHS (Arles 2007). Paris: 132-142.

Cabré Aguiló, J. 1934: "Las cuevas de los Casares y de la Hoz". Archivo Español de Arte y Arqueología X (30): 225-254.

Callejo Serrano, C. 1958: La cueva prehistórica de Maltravieso, junto a Cáceres. Publicaciones de la Biblioteca Pública de la Ciudad. Cáceres.

Corchón, M. ${ }^{a}$ S. (ed.) 1997: La cueva de La Griega de Pedraza (Segovia). Memorias Arqueología en Castilla y León 3, Junta de Castilla y León, Consejería de Cultura y Turismo. Zamora.

Corchón, M. ${ }^{\text {a }}$ S. 2006: "Reflexiones sobre el arte paleolítico interior: la meseta norte española y sus relaciones con Portugal". Zephyrus 59: 111-134.

Corchón, M. S.; Rivero, O.; Hernando, C. y Garate, D. 2012: "La cueva de La Griega (Pedraza, Segovia, España) en la encrucijada ibérica: nuevos análisis del arte parietal paleolítico a través del análisis factorial de correspondencias". Espacio, Tiempo y Forma, Nueva época, Serie i 5: 533-548. https://doi.org/10.5944/etfi.5.2012.5358

Díez, C. y Navazo, M. 2005: “Apuntes sociales y geográficos a partir de los yacimientos del Paleolítico Medio en la zona nororiental de la Meseta castellano-leonesa". En R. Montes Barquín y J. A. Lasheras (eds.): Actas de la reunión cientifica: Neandertales cantábricos. Estado de la cuestión. Monografías del Museo Nacional y Centro de Investigación de Altamira 20, Ministerio de Cultura. Madrid: 39-54.

Domingo, I.; Villaverde, V.; López Montalvo, E.; Lerma, J. L. y Cabrelles, M. 2013: "Latest developments in rock art recording: towards an integral documentation of Levantine rock art sites combining 2D and 3D recording techniques". Journal of Archaeological Science 40 (4): 1879-1889. https://doi.org/10.1016/j.jas.2012.11.024

Feruglio, V.; Dutailly, B.; Ballade, M.; Bourdier, C.; Ferrier, C.; Konik, S... y Jaubert, J. 2013: "Un outil de relevés 3D partagé en ligne: premières applications pour l'art et la taphonomie des parois ornées de la grotte de Cussac (ArTaPOC / programmeLaScArBx)". En R. Vergnieux y C. Delevoie (eds.): Virtual Retrospect (Actes de Colloque de Pessac 2013). Ausonius. Bordeaux: 49-54.

Figueiredo, S.; Nobre, L.; Ropero, A.; Xavier, P.; Gaspar, R. y Carrondo, J. 2014: "Reassembly methodology in Palaeolithic engraved plaques from Foz do Medal Terrace (Trás-os-Montes, Portugal)”. En M. A. Medina-Alcaide, A. J. Romero-Alonso, R. M. Ruiz-Márquez y J. L. Sanchidrián-Torti (eds.): Sobre rocas y huesos: las sociedades prehistóricas y sus manifestaciones plásticas. Universidad de Córdoba; Patronato de la Cueva de Nerja. Córdoba: 428-439.

Fritz, C.; Willis, M. D. y Tosello, G. 2016: "Reconstructing Paleolithic cave art: the example of Marsoulas Cave (France)". Journal of Archaeological Science: Reports 10: 910-916. https://doi.org/10.1016/j.jasrep.2016.05.012

Garate, G.; Ríos, J.; Pérez, R.; Rojas, R. y Santonja, M. 2016: “Arte rupestre Paleolítico al aire libre en el paraje de La Salud (Valle del Tormes, Salamanca)". Zephyrus LXXVII: 15-29. https://doi.org/10.14201/zephyrus2016771529

Garate, D. y Rivero, O. 2015: 'La 'Galería de los bisontes': un nuevo sector decorado en la Cueva de Alkerdi (Urdazubi/Urdax, Navarra)". Zephyrus LXXV: 17-39. https://doi.org/10.14201/zephyrus2015751739

Garate, D.; Rivero, O.; Bourrillon, R. y Pétillon, J. M. 2013: "La grotte ornée de Sainte-Colome à Arudy dans le Tardiglaciaire des Pyrénées occidentales". Paléo 24: 103-120.

Gonzalo Quintanilla, F. 1970: “Arte rupestre en la Provincia de Segovia”. Revista Ejército 370: 5-9.

Guy, E. 2002: “Contribution à la stylistique à l'estimation chronologique des piquetages paléolithiques de la vallée du Cõa (Portugal)". En D. Sacchi (ed.): Le paysage modifié par l'image. Actes du Colloque International Faculté des Lettres et Sciences Humaines de Perpignan, 
UMR 5590 du CNRS, Laboratoire de Préhistoire de Tautavel (Tautavel-Campôme 2000). Carcassonne: 65-72.

Hernández Pacheco, E. 1917: Los grabados de la cueva de Penches. Junta para la Ampliación de Estudios e Investigaciones Científicas, Comisión de Investigaciones Paleontológicas y Prehistóricas, Memoria 17, Museo Nacional de Ciencias Naturales. Madrid

Hernández Pacheco, E. 1959: Prehistoria del Solar Hispano. Orígenes del arte pictórico. Ed. Real Academia de las Ciencias Exactas, Físicas y Naturales. Madrid.

Jorge, S. O.; Jorge, V. O.; Almeida, C. A. F. de; Sanches, M. J. y Soeiro, M. T. 1981: "Gravuras rupestres de Mazouco (Freixo de Espada a Cinta)". Arqueologia 3: 312.

Jorge, S. O.; Jorge, V. O.; Almeida, C. A. F. de; Sanches, M. J. y Soeiro, T. 1982: "Descoberta de gravuras rupestres em Mazouco, Freixo de Espada à Cinta (Portugal)". Zephyrus XXXIV-XXXV: 65-70.

Lucas Pellicer, M. ${ }^{a}$ R. 1973: "Grabados rupestres de Domingo García (Segovia)". En XII Congreso Nacional de Arqueología (Jaén 1971). Zaragoza: 257-266.

Luís, L. 2012: "Desenhos animados! Uma gramática do movimento para a arte paleolítica do Vale do Côa". Trabalhos de Arqueologia 54: 6980

Martínez García, J. 1986-1987: "Un grabado paleolítico al aire libre en Piedras Blancas (Escullar, Almería)". Ars Praehistorica V-VI: 49-58.

Plisson, H. y Zotkina, L.V. 2015: "From 2D to 3D at macro- and microscopic scale in rock art studies". Digital Applications in Archaeology and Cultural Heritage 2: 102-119. https://doi.org/10.1016/j.daach.2015.06.002

Reis, M. y Vázquez, C. 2015: “Arte rupestre en la frontera hispano-portuguesa: cuenca del río Águeda”. ARPI 03 Extra: 32-43.

Ripoll, S. y Municio, L. J. (eds.) 1999: Domingo García. Arte rupestre paleolitico al aire libre en la Meseta castellana. Memorias Arqueología en Castilla y León 8, Junta de Castilla y León, Consejería de Cultura y Turismo; UNED. Salamanca.

Rivero, O. 2010: "Las manifestaciones artísticas al aire libre al sur del río Duero; los yacimientos de Foz Côa y Siega Verde en el contexto del arte paleolítico peninsular". En N. Cubas, D. Hidalgo y M. Salinas (eds.): Arqueología, Prehistoria e Historia Antigua de los pueblos "sin pasado": ecos de la Lusitania en Arribes del Duero. Colección Aquilafuente. Universidad de Salamanca. Salamanca: 83-96.

Rivero, O.; Ruiz-López, J. F.; Intxaurbe, I.; Salazar, S. y Garate, D. 2019 : "On the limits of 3D capture: a new method to approach the photogrammetric recording of palaeolithic thin incised engravings in Atxurra Cave (northern Spain)". Digital Applications in Archaeology and Cultural Heritage 14: e00106.

https://doi.org/10.1016/j.daach.2019.e00106

Robert, E.; Petrognani, S. y Lesvignes, E. 2016: "Application of digital photographyinthestudy of Paleolithic cave art". Journal of Archaeological Science: Reports 10: 847-858.

Sacchi, D.; Abelanet, J.; Brule, J. L.; Massiac, Y.; Rubiella, C. y Vilette, P. 1988: "La roche gravé de Fornols-Haut à Campome, PyrénéesOrientales, France. Étude preliminaire”. Bajo Aragón Prehistoria 7-8: 279-293.

Santos, A. T. 2019: A arte paleolítica ao ar livre da bacia do Douro à margem direita do Tejo: uma visão de conjunto. Monografias AAP 9. Associação dos Arqueólogos Portugueses. Lisboa

Sauvet, G. y Sauvet, S. 1983: Los grabados rupestres prehistóricos de la cueva de La Griega (Pedraza, Segovia). Corpus Artis Rupestris 1, Palaeolithica Ars 2, Dpto. Prehistoria y Arqueología. Salamanca.

Teixeira, J. C. y Sanches, M. J. 2017: "O abrigo rupestre da foz do Tua no contexto da Arte Paleolítica e Pós-Paleolítica". Portvgalia, Nova Série 38: 9-48. https://doi.org/10.21747/09714290/port38a1

Valladas, H.; Mercier, N.; Froget, L.; Jorons, J.-L.; Reyss, J.-L. y Aubry, T. 2001: "TL dating of Upper Palaeolithic sites in the Côa Valley (Portugal)". Quaternary Science Reviews 20: 939-943. https://doi.org/10.1016/s0277-3791(00)00026-3

Welker, W. 2014: "Felsbilder im Hunsrück Erste paläolithische Felkunst in Deutschland". En Eiszeitjäger. Leben im Paradies?: Europa vor 15.000 Jahren Gebundene Ausgabe. LVR-LandesMuseum Bonn. Bonn: 9-33. 